\title{
CARACTERIZAÇÃO DE UMA FITOALEXINA EM FOLHAS DE AMENDOIM: MEDICARPINA CONTRA A "MANCHA-PRETA"
}

\author{
Lilian C. Correia ${ }^{1}$, Vanessa G. Monteiro ${ }^{3}$, Daniela M. Soares², João Francisco dos Santos ${ }^{4}$, \\ Denizart Bolonhezi ${ }^{4}$, Alberto J. Cavalheiro ${ }^{5}$, Ignácio J. Godoy ${ }^{4}$ e Cássia R. Limonta Carvalho ${ }^{4}$
}

\begin{abstract}
${ }^{1}$ Pós-graduanda em Química, IQ/UNESP, Araraquara, SP, lilian.correia@iac.gov.br (Apresentadora do trabalho); ${ }^{2}$ Química, Centro de Recursos Genéticos Vegetais/IAC, Campinas, SP, dmatsumoto@iac.sp.gov.br; ${ }^{3}$ Pós-graduada em Agricultura Tropical e Subtropical/IAC, va.gmonteiro@gmail.com; ${ }^{4}$ Pesquisador Científico/IAC, Campinas, SP, joaofsantos@iac.sp.gov.br, denizart@iac.sp.gov.br, ijgodoy@iac.sp.gov.br, climonta@iac.sp.gov.br; ${ }^{5}$ Docente do IQ/UNESP, Araraquara, SP, albjcava@gmail.com.
\end{abstract}

RESUMO: A cultura do amendoim no Brasil tem sido bastante afetada por doenças da parte aérea, como a mancha-preta causada pelo fungo Cercosporidium personatum (Berk. \& M.A. Curtis) Deighton. Embora já se conheçam fontes de resistência a serem utilizadas nos programas de melhoramento de amendoim do Instituto Agronômico de Campinas (IAC), os mecanismos de resistência ainda não foram confirmados. De acordo com a literatura, sabe-se que um dos mecanismos de resistência das plantas é a produção de compostos provenientes do seu metabolismo secundário. Portanto, este trabalho teve como objetivo identificar e caracterizar possíveis fitoalexinas presentes nas folhas de dois cultivares contrastantes quanto à resistência a mancha-preta. Cada cultivar foi avaliado em duas condições de campo: com e sem controle químico. A avaliação metabolômica foi realizada por Cromatografia Líquida de Alta Eficiência (HPLC) acoplada a detecção de arranjo de diodos (DAD) e espectrometria de massas de alta resolução (HRMS). Os resultados obtidos mostraram o acúmulo do isoflavonóide medicarpina e maior quantidade do flavonol quercetina-3-O-glucoronídeo no cultivar resistente ao longo das coletas. Já no cultivar susceptível, esse acúmulo não foi observado, evidenciando que a resistência está associada à produção da medicarpina.

Palavras-Chave: Arachis hypogaea, mancha-preta, Cercosporidium personatum, medicarpina, quercetina-3-O-glucoronídeo, metabolômica.

\section{INTRODUÇÃO}

A cultura do amendoim apresenta muitas doenças em todo o seu ciclo, sendo as manchas foliares, ocasionadas por fungos do gênero Cercospora, as principais causadoras de prejuízos, podendo reduzir significativamente a produção de vagens e a qualidade dos grãos; perdas da produtividade dos grãos podem chegar até 60\% (GODOY et al., 2001). A principal forma de controle é a aplicação de fungicidas, mas, o uso de material genético com resistência varietal tem sido uma das 


\section{Encontro Sobre a Cultura do Amendoim \\ 15 a 17 de agosto de 2019 na Estação de Eventos Cora Coralina e FCAV/UNESP, Jaboticabal-SP}

formas mais eficazes e sustentáveis de controle dessas doenças. O cultivar Runner IAC 886 e o Acesso 69007 são considerados susceptível e resistente, respectivamente, à macha-preta ( $C$. personatum), porém o mecanismo dessa resistência ainda não foi totalmente elucidado. Portanto, o objetivo desse trabalho foi a investigação metabolômica dos extratos provenientes de folhas de amendoim em diferentes tempos de coleta para comparação entre os dois cultivares e elucidação do mecanismo químico de defesa contra o fungo causador da mancha-preta.

\section{MATERIAL E MÉTODOS}

Os genótipos utilizados neste trabalho (Tabela 1) foram coletados no Banco de Germoplasma do Programa de Melhoramento de Amendoim do IAC. O experimento de campo foi conduzido no Polo Regional Centro Leste, da APTA, em Ribeirão Preto, de janeiro à maio de 2015 e as análises químicas realizadas no Laboratório de Fitoquímica, do Centro de Recursos Genéticos Vegetais/IAC.

A área experimental foi previamente calcareada e adubada antes do plantio com 300 $\mathrm{kg} / \mathrm{hectare}$ da fórmula 4-30-10. O experimento foi semeado em esquema fatorial $8 \times 2$, em delineamento de blocos ao acaso com três repetições, em parcelas de duas linhas de plantas de 5 metros, com espaçamento de 0,9 metros entre linhas. Cada genótipo foi avaliado em duas condições: com controle químico $\left(\mathrm{C}_{\mathrm{CQ}}\right)$ e sem controle químico $\left(\mathrm{S}_{\mathrm{CQ}}\right)$. O controle químico consistiu de sete pulverizações com fungicidas, sendo seis de Chlorothalonil e uma de mistura de Epoxiconazol e Piraclostrobina durante o ciclo. As aplicações foram feitas quinzenalmente a partir de 35 dias da semeadura (DAS).

A incidência da mancha-preta foi avaliada no campo, aos 65, 94, 108, 122 e 135 DAS por 3 avaliadores, mediante o uso da escala diagramática proposta por SUBRAHMANYAM et al. (1982), em que as notas variaram de 1 (ausência de sintomas da doença) a 9 (grande número de lesões nas folhas). A partir desses dados, a área sob a curva de progresso da doença (ASCPD) foi estimada para cada dia de avaliação, de acordo com método proposto por MORAES (1987).

Para análises químicas, coletas e preparos do material vegetal dos genótipos de amendoim foram realizados nos mesmos dias das avaliações da doença no campo (Tabela 1). Vinte folíolos aleatórios, situados em posição mediana em relação à haste principal, sendo dez de cada linha, foram coletados e imediatamente transferidos para uma caixa de isopor contendo nitrogênio líquido. No laboratório, os folíolos foram moídos e $0,5 \mathrm{~g}$ de cada amostra foram extraídos com $40 \mathrm{~mL}$ de metanol:água destilada $(6: 4 ; \mathrm{v} / \mathrm{v})$. A análise dos compostos foi feita pela técnica de HPLC-DAD-MS, segundo método descrito por LIU et al. (2005). 


\section{Encontro Sobre a Cultura do Amendoim \\ 15 a 17 de agosto de 2019 na Estação de Eventos Cora Coralina e FCAV/UNESP, Jaboticabal-SP}

Tabela 1. Material genético utilizado no trabalho e datas de coleta (em DAS) para avaliação da incidência de mancha-preta e análises metabolômicas.

\begin{tabular}{cccccc}
\hline \hline Germoplasma & Origem & Subespécie & Grupo & Crescimento & Coleta (DAS) \\
\hline Acesso 69007 & ICRISAT (Índia) & hypogaea & - & Arbustivo & $65,94,108$, \\
Cultivar Runner IAC 886 & IAC & hypogaea & Virgínia & Rasteiro & $122 \mathrm{e} \mathrm{135}$ \\
\hline \hline
\end{tabular}

\section{RESULTADOS E DISCUSSÃO}

Pela incidência da mancha-preta no campo observou-se que as pulverizações foram efetivas, já que os valores de $\mathrm{ASCPD} / \mathrm{C}_{\mathrm{CQ}}$ se mantiveram baixos (Figura 1A). Também foi possível observar diferenças no comportamento dos genótipos em relação ao desenvolvimento da doença (Figura 1B). As taxas médias de progressão da doença, expressas em ASCPD, foram de 1,8 e 1,4 para os genótipos 886 e 69007, respectivamente. No primeiro estágio, entre 65 e 94 DAS, período em que as condições de temperatura e umidade foram favoráveis para o desenvolvimento do fungo, a incidência da doença foi aproximadamente $30 \%$ maior no cultivar 886 comparado ao 69007. Entre os 94 e 108 DAS, o '886' apresentou 80 \% a mais de lesões nas folhas. Entre 108 e 122 DAS, a média da ASCPD do '886' foi $100 \%$ maior em relação à média do '69007'. E no último período, de 122 a 135 DAS, as plantas do '886' encontravam-se totalmente desfolhadas. A partir dos dados apresentados comprovou-se a condição de suscetibilidade da cultivar IAC 886 em relação à incidência da mancha-preta, uma vez que apresentou valores de ASCPD, para o tratamento $\mathrm{S}_{\mathrm{CQ}}$ e em cada DAS, significativamente mais elevados do que o acesso 69007, como também diferenças significativas das médias entre cada DAS.
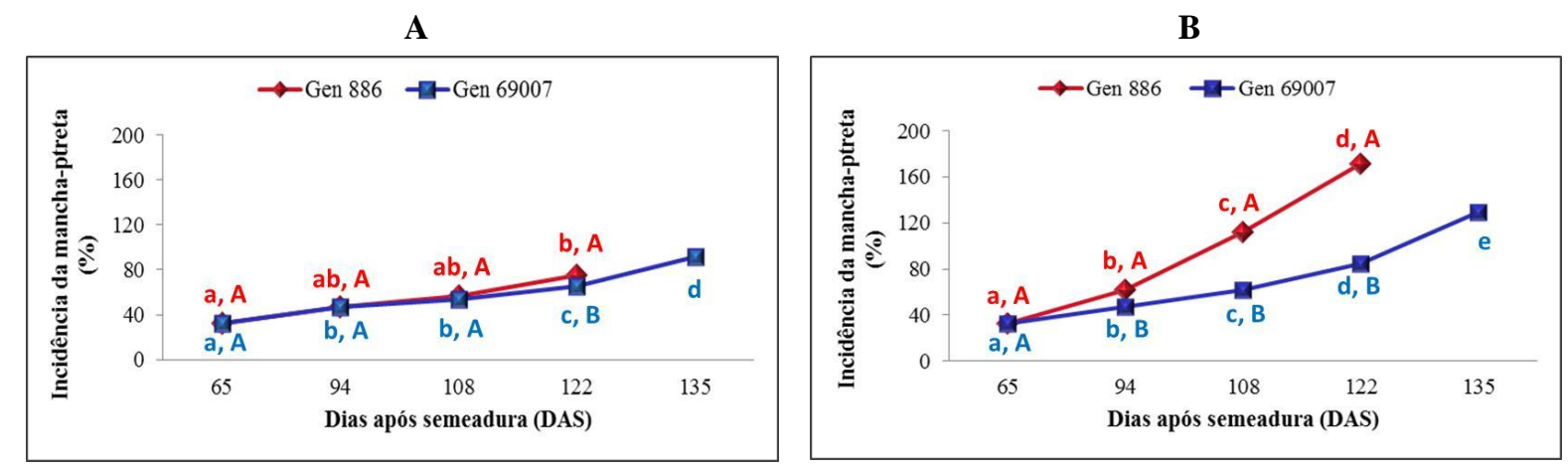

Figura 1. Curvas de incidência de mancha-preta expressas em ASCPD (\%) e avaliadas em campo em diferentes DAS, para os tratamentos: A) $\mathrm{C}_{\mathrm{CQ}}$ e B) $\mathrm{S}_{\mathrm{CQ}}$. As letras acima de cada ponto representam o teste de Tukey a 5\% de probabilidade - em vermelho para genótipo 886 e em azul para 69007. Letras minúsculas representam as diferenças entre DAS e as letras maiúsculas representam diferenças entre os genótipos em cada DAS. Pontos seguidos de mesma letra não diferem entre si.

Durante a análise metabolômica foi observado o aparecimento e acúmulo de uma substância no cultivar resistente/tratamento $\mathrm{S}_{\mathrm{CQ}}$, ao longo das coletas (Figura 2). Os cromatogramas em azul e vermelho representam a $1^{\mathrm{a}}$ e $2^{\mathrm{a}}$ coletas dos folíolos, respectivamente, aos 65 e 94 DAS, onde o pico 


\section{Encontro Sobre a Cultura do Amendoim \\ 15 a 17 de agosto de 2019 na Estação de Eventos Cora Coralina e FCAV/UNESP, Jaboticabal-SP}

em 53,3 min não aparece; a substância revelou-se aos 108 DAS e, a partir desta data, se intensificou, com área/mg amostra 22,1 vezes maior aos 135 DAS. Entre o conjunto de compostos pré-formados nos folíolos, o pico a 23,3 min foi o majoritário. Posteriormente, os extratos foram analisados em um UPLC-QTOF-MS/MS a fim de se identificar esses compostos, por meio da massa de alta resolução, fragmentos e comparação com a literatura. No tempo de retenção 53,3 min observou-se a presença de $m / z$ 269,0820 [M-H] correspondente ao isoflavonóide medicarpina ( $\Delta \mathrm{m}=2,6 \mathrm{ppm}$ ), que se correlacionou significativamente com as avaliações ASCPD ( $r=0,956 ; p<0,01)$. Em 23,3 min observou-se a presença de $\mathrm{m} / z$ 477,0669 $[\mathrm{M}-\mathrm{H}]^{-}$correspondente à quercetina-3-O-glucoronídeo $(\Delta \mathrm{m}=$ 0,6 ppm), embora majoritária entre os compostos constituintes dos extratos, não se correlacionou com as avaliações ASCPD, permanecendo estável durante o crescimento das plantas. Para os cromatogramas do cultivar susceptível $\left(886 / \mathrm{S}_{\mathrm{CQ}}\right)$, o aparecimento de uma nova substância ao longo do tempo não foi observada. Foram detectados os compostos pré-formados, predominando a quercetina3-O-glucoronídeo presente em quantidades 3 vezes menores quando comparada ao genótipo 69007.

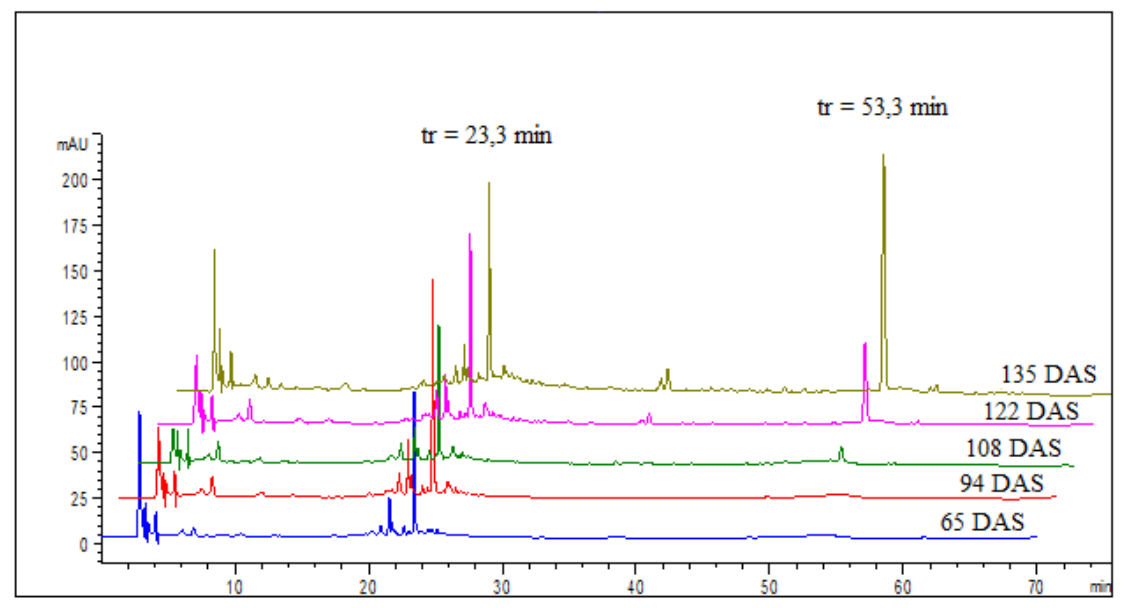

Figura 2. Cromatogramas obtidos por HPLC-DAD, em 275 nm, dos extratos metanólicos do genótipo resistente $\left(69007 / \mathrm{S}_{\mathrm{CQ}}\right)$, plotados para cada DAS.

A Tabela 2 mostra a presença ou ausência da medicarpina ao longo das coletas e observa-se uma notável relação entre a produção dessa substância e o aparecimento da mancha-preta a partir da expressão máxima da doença (108 DAS) nas folhas no tratamento $\mathrm{S}_{\mathrm{CQ}}$. Portanto, correlaciona-se a resistência do cultivar 69007 com a capacidade de produção da medicarpina. 
XVI Encontro Sobre a Cultura do Amendoim

15 a 17 de agosto de 2019 na Estação de Eventos Cora Coralina e FCAV/UNESP, Jaboticabal-SP

Tabela 2. Presença do isoflavonóide medicarpina ao longo do tempo nos germoplasmas controle susceptível (IAC 886) e resistente (69007). $\mathrm{C}_{\mathrm{CQ}}$ : controle químico e $\mathrm{S}_{\mathrm{CQ}}$ : sem controle químico.

\begin{tabular}{ccccccccccc}
\hline \hline & \multicolumn{10}{c}{ Coleta (DAS) } \\
\cline { 2 - 10 } Germoplasma & \multicolumn{6}{c}{$\mathbf{6 5}$} & $\mathbf{9 4}$ & \multicolumn{10}{c}{$\mathbf{1 0 8}$} & $\mathbf{1 2 2}$ & $\mathbf{1 3 5}$ \\
\cline { 2 - 10 } & $\mathrm{C}_{\mathrm{CQ}}$ & $\mathrm{S}_{\mathrm{CQ}}$ & $\mathrm{C}_{\mathrm{CQ}}$ & $\mathrm{S}_{\mathrm{CQ}}$ & $\mathrm{C}_{\mathrm{CQ}}$ & $\mathrm{S}_{\mathrm{CQ}}$ & $\mathrm{C}_{\mathrm{CQ}}$ & $\mathrm{S}_{\mathrm{CQ}}$ & $\mathrm{C}_{\mathrm{CQ}}$ & $\mathrm{S}_{\mathrm{CQ}}$ \\
\hline Acesso 69007 & - & - & - & - & - & $\mathrm{Sim}$ & - & $\mathrm{Sim}$ & $\mathrm{Sim}$ & $\mathrm{Sim}$ \\
Cultivar Runner IAC 886 & - & - & - & - & - & - & - & - & - & - \\
\hline \hline
\end{tabular}

A medicarpina, representada na Figura 3A, é considerada uma fitoalexina, ou seja, um metabólito antimicrobiano produzido por plantas em resposta ao contato com parasitas. Diversos trabalhos relatam a medicarpina como fitoalexina formada em leguminosas, entre eles sua presença em folhas de amendoim contra Cercospora arachidicola (mancha-castanha), Phoma arachidicola (mancha-barrenta), Puccinia arachidis (ferrugem) e Helminthosporium Carbonum (EDWARDS et al., 1995; STRANGE et al.,1985). Porém, até onde se tem conhecimento, o presente trabalho confirmou a presença da medicarpina descrita no único trabalho a correlacionar essa fitoalexina com o fungo Cercosporidium personatum (Berk. \& M.A. Curtis) Deighton, causador da mancha-preta nas folhagens de amendoim (RAO et al. 1996).

A

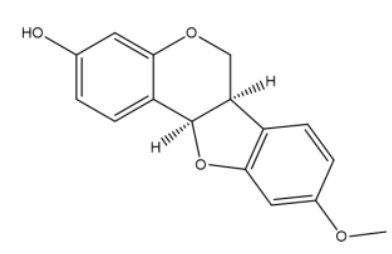

B

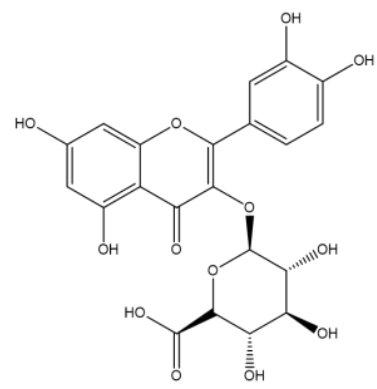

Figura 3. A) Estruturas químicas da medicarpina e B) da quercetina-3-O-glucoronídeo.

\section{CONCLUSÕES}

A resistência do cultivar 69007 à mancha-preta foi associada ao acúmulo da fitoalexina medicarpina e maior quantidade da quercetina-3-O-glucoronídeo. A quantificação do teor desses metabólitos está em andamento e os resultados serão publicados futuramente.

\section{AGRADECIMENTOS}

Os autores agradecem ao Centro de Cana/IAC, em Ribeirão Preto, SP, pelo empréstimo de laboratório e equipamentos na etapa de preparação do material vegetal para as análises químicas. 


\section{Encontro Sobre a Cultura do Amendoim}

15 a 17 de agosto de 2019 na Estação de Eventos Cora Coralina e FCAV/UNESP, Jaboticabal-SP

\section{REFERENCIAS BIBLIOGRÁFICAS}

EDWARDS, C.; STRANGE, R.N.; COLE, D.L. Accumulation of isoflavonoid phytoalexins in leaves of Arachis hypogaea differing in reaction to rust (Puccinia arachidis) and early leafspot (Cercospora arachidicola). Plant pathology, v. 44, n. 3, p. 573-579, 1995.

GODOY, I.J.; MORAES, S.A.; MORAES, A.R.A.; KASAI, F.S.; MARTINS, L.M.; PEREIRA, J.C. V.N.A. Potencial produtivo de linhagens de amendoim do grupo ereto precoce com e sem controle de doenças foliares. Bragantia, v. 60, n. 2, p. 101-110, 2001.

LIU, RONG-XIA; WANG, Q.; GUO, H.Z.; LI, L.; BI, K.S.; GUO, D. A. Simultaneous determination of 10 major flavonoids in Dalbergia odorifera by high performance liquid chromatography. Journal of pharmaceutical and biomedical analysis, v. 39, n. 3-4, p. 469-476, 2005.

MORAES, S. A. Escalas diagramáticas para avaliar a intensidade das cercosporioses do amendoim. $\mathbf{O}$ Agronômico, Campinas, v. 39, p. 9-18, 1987.

RAO, P.V.S; WADIA, K.D.R.; STRANGE, R.N. Biotic and abiotic elicitation of phytoalexins in leaves of groundnut (Arachis hypogaea L.). Physiological and molecular plant pathology, v. 49, n. 5, p. 343-357, 1996.

STRANGE, R.N.; INGHAM, J.L.; COLE, D.L.; CAVILL, M.E.; EDWARDS, C.; COOKSEY, C.J.; GARRATTD, P.J. Isolation of the phytoalexin medicarpin from leaflets of Arachis hypogaea and related species of the tribe Aeschynomeneae. Zeitschrift für Naturforschung C, v.40, n. 5-6, p. 313316, 1985.

SUBRAHMANYAM, P.; McDONALD, D.; GIBBONS. R.W.; NIGAM, S.N.; NEVILL, D.J. Resistance to rust and late leaf spot diseases in some genotypes of Arachis hypogaea. Peanut Science, v. 9, p. 6-10, 1982. 